\title{
Motivation and learning styles in young children with Down syndrome
}

\author{
Jennifer Wishart \\ Moray House Institute of Education, University of Edinburgh, Scotland, UK
}

\begin{abstract}
There are both psychological and biological reasons to expect that certain areas of learning will present young children with Down syndrome with significant problems. Knowledge of the neurological underpinnings of these specific difficulties can often allow compensatory teaching strategies to be put in place, however, and some of these have proved highly effective. The impact of the psychological environment on the progress of development in children with Down syndrome is less well understood. Experience of how others respond to their attempts at understanding the physical and social world and the balance of successes and failures they experience in their early learning are both likely to influence the approach to learning adopted when faced with mastering new skills. Findings from inter-linking studies of cognitive and socio-cognitive development which have explored learning behaviours at different ages and at different developmental stages illustrate how a learning style can sometimes evolve over time in which less than efficient use is made of current levels of cognitive ability. Social ploys are sometimes used to avoid engagement in learning, with the net effect that opportunities to learn new skills are not fully exploited and old skills are sometimes inadequately consolidated. Findings of a misuse of social skills in cognitive contexts do not necessarily provide support for the widely-held view that social understanding is an area of strength in children with Down syndrome and less vulnerable to disruption than cognitive development. Data from a recent study of face-processing abilities suggest that there may in fact be a specific weakness in a fundamental skill normally underpinning the development of social understanding: the ability to recognise differences in emotional expressions. The children with Down syndrome in this study had few problems in correctly identifying individual faces but evidenced difficulties in reliably interpreting the emotional expressions portrayed in these faces. These findings are consistent with the emerging picture of neurological disruption in Down syndrome and with what is known of the neurology underlying this key component in social cognition. As most learning takes place in a social context, the findings have implications for adult-child and child-child learning partnerships and would seem to merit further investigation.
\end{abstract}

Keywords - learning style, social cognition, neuropsychology, collaborative learning

\section{Introduction}

Learning in children with Down syndrome is inevitably greatly compromised by the disruption to developmental processes caused by the presence of the extra copy of chromosome 21. Differences in the structure and development of the brain mean that a number of basic information processing skills central to the achievement of specific cognitive and linguistic milestones are adversely affected. Research over the last two decades has greatly increased our knowledge of the specific difficulties associated with the syndrome, however, and this has allowed compensatory teaching strategies to be put in place, some of which have proved highly effective (see e.g. the work of Sue
Buckley and her team - forthcoming issue of Down Syndrome Research \& Practice.

If teaching methods are to ensure that children with Down syndrome derive maximum benefits from their inherent and acquired abilities, it is important that we recognise both the strengths and weaknesses in their cognitive profiles, using the former to compensate for the latter wherever possible. It is also important that we recognise that the children's motivation to learn may be undermined by their less than favourable experiences of learning if this is not carefully monitored and managed.

This article presents an overview of findings from a number of studies of cognitive development in children with Down syndrome carried out in Edinburgh over the last ten years 
or so. Studies are presented in outline only and readers are referred to the original articles for more detailed information. Space restrictions also dictate that no attempt can be made here to review related work by other research teams working in the area of motivation and learning in children with Down syndrome, some of which reflects contrary views to those expressed here. Key contributions by other research teams are discussed in some of the articles and chapters referenced at the end of this paper, however, and those interested in delving deeper into the literature associated with the themes explored in this article are directed to these and the original sources.

\section{Development in children with Down syndrome}

Researchers are still divided over whether development in children with Down syndrome is best understood in terms of a slowed-down version of typical development or whether it is fundamentally different in its nature and progression. Linked to the assumption of slowed-down typical development - and to terms such as 'mental retardation', 'developmentally delayed' and 'slow learner' - is the assumption that a fuller understanding of development in Down syndrome will follow from an increased understanding of factors influencing developmental progression in typically developing children.

Both assumptions seem unsafe for a number of reasons, not least because children with Down syndrome come to learning with a totally different set of biologically-determined 'tools' for learning to those held by typically developing children. Research has demonstrated that abilities in different developmental domains do not emerge in Down syndrome in the same synchronous manner as in typical development, with language development, for instance, usually lagging behind other areas of cognitive development. Within some specific domains (e.g. object concept development), achievement of stages also does not appear to follow the typical sequence of acquisition. It would be surprising if such asynchronies in development did not have a significant impact on developmental pathways and developmental rates, putting comparisons with typical development on a very risky footing.

In considering how development in children with Down syndrome might progress, the other factor which needs to be taken into account is the effect of the success/failure histories likely to be associated with the level of learning difficulties experienced, that is, the psychological impact of exposure to significant learning difficulties throughout life. Many of the standard skills of childhood are eventually achieved by children with Down syndrome, albeit often much later than in ordinary children. It therefore seems reasonable to suggest that for many, the motivation to invest continuing effort in learning may to some extent be put 'at risk' by their less-than-favourable learning histories. Initial failure rates are likely to be higher than for typically developing children, for example, and time taken to acquire many key skills is considerably longer, extended by years rather than months in some areas of learning. Language and communication problems associated with the syndrome can only exacerbate these difficulties in learning contexts, again a factor which might be expected to erode motivation to engage fully in learning, particularly learning alongside others. It would indeed be surprising if such a combination of factors did not progressively discourage self-generated learning activity and adversely influence the children's perceptions of themselves as learners.

\section{Children's learning partners: Attitudes and expectations}

It is also now evident that the expectations of potential for learning in children with Down syndrome are often unnecessarily low in their partners in learning, something which the children themselves often seem to pick up on and exploit (see below)

Much of the blame for this must lie with the well-established stereotype of children with Down syndrome. They are widely viewed as children who are not too bright but nevertheless happy and docile, who love music and are very affectionate, and who fit easily into family life. This is a predominantly positive stereotype but it is not a very helpful one. It is, moreover, based on very little in the way of scientific evidence. Few studies in this area have looked directly at personality characteristics in children with Down syndrome. Most simply ask others - teachers, parents or carers - to list the personality characteristics common to those with Down syndrome, usually by picking from a predetermined list of descriptors. As one of our own studies has shown, all this approach demonstrates is that the stereotype is alive and well, sometimes in some very surprising places (Wishart \& Johnston, 1990).

Our stereotyping study used a questionnaire based on the literature describing personality in children and adults with Down syndrome. We asked nine groups of adults with differing levels of experience and contact with children with Down syndrome - parents, teachers and students at a late stage in their professional training as doctors, health visitors and psychologists - to fill in a questionnaire telling us what they knew about Down syndrome as a condition and what they knew about children who had Down syndrome. They were asked to pick from a 5 point bipolar rating scale those characteristics which they 'believed best describe the personality of children with Down syndrome under 12 years of age'. The list contained 26 characteristics compiled from the literature on Down syndrome (e.g. outgoing/withdrawn; affectionate/undemonstrative); 3 of the 26 characteristics had 'ends' both of which had been described in the literature as stereotypical (e.g. docile/ stubborn).

The task set was, of course, a trick one - there is no such thing as 'the' child with Down syndrome, whatever the age - but that did not stop almost all of the sample from ticking one or other end of each of the paired characteristics. Statements like "the children are super to work with - such loving wee souls" were also readily entered in the box left 
for additional comments at the end of the questionnaire. Only five highly experienced special school teachers irately returned the questionnaire and said it was impossible to fill in meaningfully. Mainstream primary teachers, teachers from integrated schools with and without direct experience of working with children with Down syndrome, and the remaining special school teachers all returned completed questionnaires, as did the medical students, the psychology students and the health visitor trainees, all producing profiles which fitted well with the Down syndrome stereotype as portrayed in the literature.

Surprisingly, mothers who had a child with Down syndrome also readily endorsed the stereotype, although to a lesser degree. Interestingly, and paradoxically, when given the opportunity to fill in two questionnaires, one for children with Down syndrome in general and one specifically in relation to their own child with Down syndrome, they attributed the stereotype more strongly to their own child. Mothers with children of a similar age, but without Down syndrome, were also happy to endorse the Down syndrome stereotype; when asked to fill in a second questionnaire in relation to typically developing 12 year olds, however, all considered this an impossible task.

Given the major role teachers play in supporting learning, we were particularly interested in exploring attitudes and expectations in this group further. Full details of the findings of this study can be found in Wishart and Manning (1996) and are reported only in outline here. They were no more encouraging, however, than the findings from the stereotyping study. Over 200 trainee teachers from two major UK teacher education colleges were surveyed. Their views on inclusive education were solicited, along with their estimates of the ages at which they believed a number of key developmental milestones might be achieved by those with Down syndrome, from toilet-training to marriage. We also asked them how much personal contact they had had, socially or professionally, with anyone who had Down syndrome.

Although most respondents knew many of the basic facts about Down syndrome, their levels of personal experience with anyone with the syndrome were remarkably low, suggesting that social and educational inclusion is not as widespread as is commonly assumed. Only $13 \%$ of the group looked forward to teaching in inclusive settings and 96\% felt that their training had not prepared them sufficiently for meeting the special educational needs of children with Down syndrome. The majority significantly overestimated the ages at which key skills might be achieved and, most worryingly, a significant number thought life expectancy for those with Down syndrome was only 30 years, a belief likely to influence the skills they would consider relevant if teaching someone with Down syndrome. Most believed inclusive education to be more beneficial than detrimental for children with Down syndrome but saw the likely benefits being in the area of socio-emotional development rather than educational achievement.

\section{Learning styles in children with Down syndrome}

It is clear from the above that many of the adults who play a significant part in supporting the learning of children with Down syndrome may have somewhat stereotyped notions of what the children are like and can have fairly low expectations of their developmental potential. In another strand of our research investigations, we have looked at the problem from the other side of the partnership, trying to explore how the presence of Down syndrome affects the children's natural learning style and whether there are changes in this over time, as their experience of learning grows.

We were interested in exploring the strategies the children used when faced with new learning, how they responded to their mistakes, and what use they made of their potential partners in learning. We were especially interested in whether children with Down syndrome were possibly adding to their already-existing difficulties by adopting counterproductive learning strategies. If so, this might help to explain the overall decline in developmental rate that characterises post-infancy years, despite the fact that new skills are being regularly added to the repertoire. We already held some data which suggested that over time some children changed from being active and relatively able problem solvers into progressively more reluctant learners as they grew older, and it seemed that motivational deficits were possibly undermining the progress of their development.

Converging evidence from a number of studies is overviewed in Wishart (1996). These indicated that with increasing age and experience, a learning style may emerge in children with Down syndrome in which there is an increasing reliance on the help of others, even when help is not needed. This was associated with a decrease in willingness to take the initiative in problem-solving contexts. Our studies used a variety of experimental paradigms, from Piagetian search tasks, through operant conditioning tasks, to perceptual matching tasks. Despite the very different task demands of these contrasting experimental paradigms, there was a very marked similarity in some of the behaviours produced by the children, all of them reflecting a tendency to avoid engaging with tasks which were 'hard' in terms of the child's current level of cognitive ability. Children simply opted out of these tasks, refusing to engage for more than a very short time and sometimes using quite subtle social ploys to distract attention away to some lessdemanding activity, such as pretending to be very interested in something else, producing some sort of 'party-trick', or simply turning on the charm. It seemed that they preferred to avoid making errors than to attempt solving a problem which they saw as potentially difficult.

It would be easy to write off such behaviours as simple lack of co-operation or to describe the child as untestable but that would tell us little about the true level of their understanding or about the nature of their difficulties. While it can be very difficult to establish the precise level of ability in a child with Down syndrome, we can learn a great 
deal from carefully observing those situations in which they choose to engage fully in learning and those in which they opt out. Within any one child, the learning strategy adopted may be different in relation to different tasks at the same age, depending on the developmental difficulty of the task in question at that stage in the child's development. In general though, the evidence seems to support the view that the 'natural' learning style of children with Down syndrome is characterised by:

- an increasing use of avoidance strategies when faced with learning new skills

- a growing reluctance to take the initiative in learning contexts

- an over-dependence on/misuse of social skills in cognitive contexts.

If left to run its course, this counterproductive learning style could clearly have a very negative effect on the progress of learning, depriving the children of many opportunities to broaden their skills and expand their learning.

The misuse of social skills in cognitive contexts is a particularly worrying trend and it was striking how well some of the avoidance behaviours produced in response to our tasks matched the 'social' stereotype of Down syndrome. The behaviours might perhaps have been interpreted as evidence that in comparison to cognitive development, social development is an area of comparative strength in Down syndrome. The highly social behaviours seen in task settings were being produced at very specific times, however, when things were getting difficult for the child. All of the social ploys used in attempts to avoid learning were behaviours also found in typically developing young children but the difference lay in the use to which they were being put. The typically developing children we tested were equally sociable and also initiated social interactions with the tester. They tended to restrict this to between trials, however. They also worked hard at all levels of the tasks, regardless of whether these were above or below their current developmental level in terms of difficulty, with losses of attention usually short and re-engagement seldom difficult to obtain.

Findings from one of our most recent studies offer some further weight to the suggestion that social understanding is not necessarily an area of comparative strength in children with Down syndrome. In this study (Pitcairn \& Wishart, 2000; Wishart \& Pitcairn, 2000), children with Down syndrome aged 8-14 years, similarly-aged children with non-specific development delay, and typically developing children aged 3-5 years were tested for their ability to recognise individual faces and to identify the emotional expressions they portrayed. Both abilities are key perceptual competencies, central to understanding the social world in which we live and in the processing of socially transmitted information.

The tasks we used were face-matching tasks. Task demands were kept very simple, requiring minimal language comprehension, and with choice indicated simply by pointing.
The target face also remained in view throughout in order to minimise memory demands. In the identity task, photographs of 3 faces - 2 of the same individual, but with each face portraying a different emotion - were used. The child was shown a photograph and told the following: "This is a picture of Laura." Two further photographs were placed underneath, and the child then asked: "Can you show me another picture of Laura?" In the expression matching task, 3 different faces were used, two with same expression. The child was told: "This is a picture of Jonathan. Jonathan looks surprised, doesn't he?" Again, two more pictures were then placed underneath and the child asked: "Can you find me another picture of a man that looks surprised?" The emotional expressions tested were happiness, surprise, sadness, fear, anger, and disgust (with 'cross', 'scared' and 'yukky' also used to describe the angry, frightened and disgusted expressions).

It was clear from the patterns of responses within and across groups that the children with Down syndrome did not have a basic problem in processing faces per se as they proved equally proficient in identifying and distinguishing individual faces. They did, however, seem to have a specific difficulty in decoding some emotional expressions, particularly surprise and fear, difficulties that were not shared with children with a similar level of developmental delay or with typically developing children of younger ages. That this was not due to a general cognitive deficit was demonstrated by their performance on another task which was cognitively more difficult, recognising faces and identifying expressions when the faces had been rotated so as to be upsidedown or on their side. Here, their performance was no more affected by the increased difficulty than in the other two groups of children.

Two different hypotheses could explain the difficulties shown in labelling expressions of fear and surprise. Firstly, it could be that children with Down syndrome are exposed to a predominantly positive social environment and learn only to deal with positive expressions. However, the children with non-specific learning disorders, who would surely be expected to be similarly protected, showed no similar problems. The difficulty was in any case in processing both fear, a negative emotion, and surprise, a positive one, and there was no great difficulty shown with the negative emotions of anger and disgust. The second hypothesis is that the difficulties stem directly from the syndrome, a symptom of the neurological differences known to be associated with Down syndrome. This explanation would be consistent with the emerging picture of neurological disruption in Down syndrome and with what is already known about the neurological underpinnings of successful face-processing. Given this, it would appear that this is an area that might well be worth further investigation, particularly given the centrality of non-verbal communication to much of learning. 


\section{Acknowledgements}

Thanks are extended to the children, families and others who participated in the research reported above, and to the following students and colleagues in the Psychology Department, University of Edinburgh, who contributed greatly to the various studies: Louise Duffy, Alison Hamilton, Christine Johnstone, Sarah Landew, Fiona Johnston, Gayle Manning, and Tom Pitcairn. Thanks are also extended to the Medical Research Council for their continuing support of this programme of research.

\section{Correspondence}

Professor Jennifer Wishart - Moray House Institute of Education, Faculty of Education, University of Edinburgh, Holyrood Road, Edinburgh, EH8 8AQ, Scotland, UK.

-E-mail: J.Wishart@ed.ac.uk.

\section{References}

Pitcairn, T.K. \& Wishart, J.G. (2000). Face processing in children with Down syndrome. In D. Weeks, R. Chua \& D. Elliott (Eds.), Perceptual-Motor Development in Down Syndrome (pp. 123-147). Champaign, Ill.: Human Kinetics.

Wishart, J.G. (1996). Avoidant learning styles and cognitive development in young children with Down syndrome. In B. Stratford \& P. Gunn (Eds.), New Approaches to Down Syndrome (pp. 173-205). London: Cassell.

Wishart, J.G. \& Johnston, F. (1990). The effects of experience on attribution of a stereotyped personality to children with Down's syndrome. Journal of Mental Deficiency Research, 34, 409-420.

Wishart, J.G. \& Manning, G. (1996). Trainee teachers' attitudes to inclusive education for children with Down's syndrome. Journal of Intellectual Disability Research, 40, 56-65.

Wishart, J.G. \& Pitcairn, T.K. (2000). The recognition of identity and expression in faces by children with Down syndrome. American Journal on Mental Retardation, $105,466-479$. 